\title{
Exploring intrinsic MR signal relaxation in acute RF ablation lesions using T2 mapping and IR-SSFP CINE imaging
}

\author{
Venkat Ramanan ${ }^{1 *}$, Haydar Celik', Sudip Ghate ${ }^{1}$, Jennifer Barry ${ }^{1}$, Nilesh R Ghugre ${ }^{1}$, Vivian Leber ${ }^{1}$, Jeff A Stainsby ${ }^{3}$, \\ Andriy Shmatukha ${ }^{3}$, Mohammed Shurrab², Eugene Crystall ${ }^{2}$, Graham Wright $^{1}$
}

From 16th Annual SCMR Scientific Sessions

San Francisco, CA, USA. 31 January - 3 February 2013

\section{Background}

Cardiac MR has been used successfully in RF ablation therapies for arrhythmias, both for procedural planning and for post-ablation lesion imaging. Non-enhanced imaging, though it has a lower SNR, has advantages over Gdenhanced techniques mainly because contrast kinetics and dosage issues are avoided. Previously T2-weighted imaging was found to be more sensitive than T1-weighted imaging [1]. In this study, we performed non-enhanced T2 mapping and an inversion-prepared SSFP CINE imaging to characterize intrinsic relaxation behavior in acute lesions.

\section{Methods}

Using approved animal protocols, 15 ablation lesions were created using irrigated catheters, on the endocardium of the left ventricle in 8 healthy pig models under X-ray and Carto-XP guidance. After transferring the animal to the MR suite, we performed T2 mapping using a multi-echo Fast-Spin-Echo (MEFSE) sequence [2] and $\mathrm{T}^{*}$ characterization using a 2RR-IR-SSFP CINE method [3]. Both MEFSE and IRSSFP were done within 30-60 mins of creating the lesions. Then Gd-DTPA (Magnevist, $0.2 \mathrm{mmol} / \mathrm{kg}$ ) was injected and late-Gd-enhancement (LGE) scans were done multiple times from 10-60 min after contrast injection. The animals were then sacrificed and the hearts were preserved for pathology.

MEFSE images were fitted using a standard 2-parameter fit for M0 and T2. The M0 term here is a function of both true $\mathrm{M} 0$ and $\mathrm{T} 1$, because it was a single- $\mathrm{RR}(\mathrm{RR}=700 \mathrm{~ms})$ sequence. IRSSFP signal recovery is determined by $\mathrm{T} 1$ * (dependent on both $\mathrm{T} 1$ and T2). Ablation extent was confirmed by LGE and pathology. The T2 and M0 maps and IRSSFP images were then analyzed using an ROI based analysis to compare the signal in 3 zones (ablation lesion, adjacent edema and remote tissue).

\section{Results}

The T2 and relative M0 values are given in Table 1. On T2-maps there was a broad area of increased T2 in and around the ablation, likely due to edema; this area was much larger than lesion extent on pathology [4]. However the M0-maps seem to correspond closely with the actual border of ablation. Contrast in T1*-weighted IRSSFP images also clearly depicted the actual border of the ablation seen on pathology [4]. T1* is about $315 \mathrm{~ms}$ in the remote zone while in ablation zone it was significantly lower at $270 \mathrm{~ms}$.

\section{Conclusions}

The M0 and T2 maps appear to depict actual lesion area and edema respectively. T1*-weighted IRSSFP generally gives more robust visualization of the ablation lesion within a breath-hold. The high signal of the lesion core in T1 and T1*-weighted images might be due to methemoglobin, which is created upon heating blood [5]. In summary, T1-weighting seems to delineate the acute lesion core while $\mathrm{T} 2$-weighting seems to depict the overlying edema.

\section{Funding}

We gratefully acknowledge support from GE Healthcare, the Ontario Research Fund, and Canadian Institutes of Health Research. 
Table $1 \mathrm{~T} 2$ and M0 in remote, edema and ablation zones.

\begin{tabular}{ccc}
\hline $\begin{array}{c}\text { Area of } \\
\text { myocardium }\end{array}$ & $\begin{array}{c}\text { T2 in ms: Mean } \\
\text { (Range) }\end{array}$ & $\begin{array}{c}\text { M0 map: Percentage increase of mean signal of ROI compared to remote zone. [ 100*(ROI- ROI_remote)/ } \\
\text { ROI_remote ] }\end{array}$ \\
\hline Remote zone & $47(42-53)$ & $0 \%$ \\
Edema zone & $79(65-103)$ & $31 \%$ \\
Ablation zone & $83(69-98)$ & $85 \%$ \\
\hline
\end{tabular}

The table shows the values of $\mathrm{T} 2$ and $\mathrm{M0}$ in the ablation, edema and remote zones. The ablation zone was defined as the area of high signal intensity on MOmap near the ablation as confirmed by LGE and pathology. The edema zone was defined as the area of high T2 values near the ablation. It surrounds the ablation zone. The remote zone was defined on the same slice far from the ablation. Note that in the ablation zone, M0 increased by $85 \%$, in the edema zone about 30\%. The T2 values increased in the ablation and edema zones by about the same amount (30-35 ms).

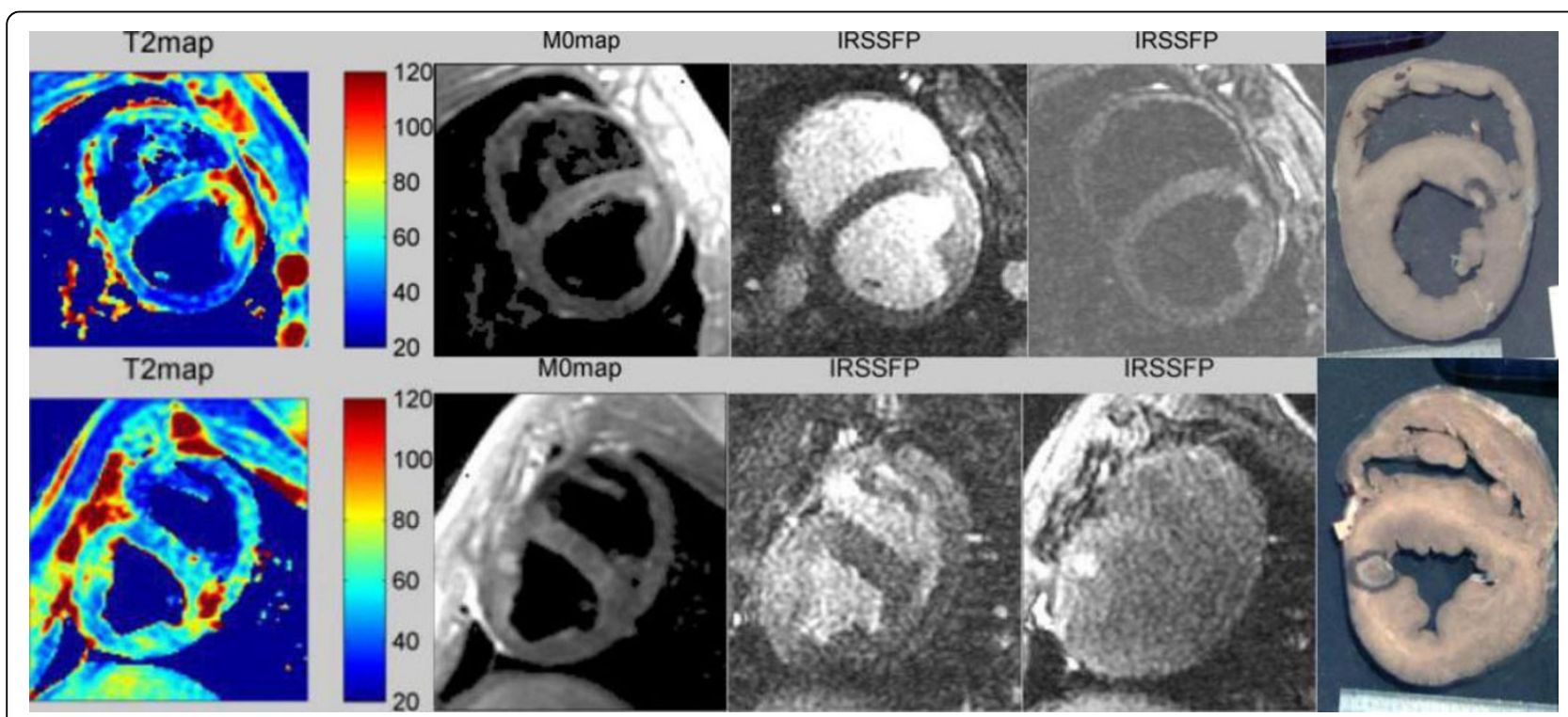

Figure 1 The two rows show ablations in two pigs. The first two columns show T2maps and MOmaps fitted from T2-mapping MEFSE sequence. The next two show IR-SSFP CINE images for two phases with different inversion times and the last one shows gross pathology of the lesion. The T2maps show a large area of diffuse T2 enhancement in and around the ablation region possibly due to edema. The M0 maps closely correspond in size and location to the ablation seen in gross pathology. Note that the MOmap is T1-weighted. IRSSFP shows two different heartphases from CINE reconstruction. The first phase is acquired in the first-RR with a short inversion time (Tl=116 ms) and therefore has bright blood while the second phase is acquired later in the second-RR $(T \mid=616 \mathrm{~ms})$ when the blood signal is nulled. Signal enhancement around the ablation region in the first phase corresponds to edema and in the second phase to the actual border.

\section{Author details}

'Imaging Research, Sunnybrook Research Institute, Toronto, ON, Canada.

${ }^{2}$ Arrhythmia Services, Sunnybrook Health Sciences Centre, Toronto, ON,

Canada. ${ }^{3}$ GE Healthcare, Toronto, ON, Canada.

Published: 30 January 2013

\section{References}

1. Dickfeld, et al:., doi:10.1016/j.hrthm.2006.10.019.

2. Stainsby, et al:. JCMR 2010, 12(Suppl 1):P227.

3. Detsky, et al: MRM 2007, 58:365-372.

4. Celik, et al:. SCMR 2013, (Submitted).

5. Farahani, et al:. MRI 1999, 17(10):1489-1494.

doi:10.1186/1532-429X-15-S1-087

Cite this article as: Ramanan et al:: Exploring intrinsic MR signal relaxation in acute RF ablation lesions using T2 mapping and IR-SSFP CINE imaging. Journal of Cardiovascular Magnetic Resonance 2013 15(Suppl 1):087.

\section{Submit your next manuscript to BioMed Central and take full advantage of:}

- Convenient online submission

- Thorough peer review

- No space constraints or color figure charges

- Immediate publication on acceptance

- Inclusion in PubMed, CAS, Scopus and Google Scholar

- Research which is freely available for redistribution 